\title{
Spectral-Luminescent and Electroluminescent Properties of Charge- Transfer Systems Based on Electron-Donating Diphenylamine Derivatives and Acceptors of Dibenzothiophene Sulfone and Phenanthridine
}

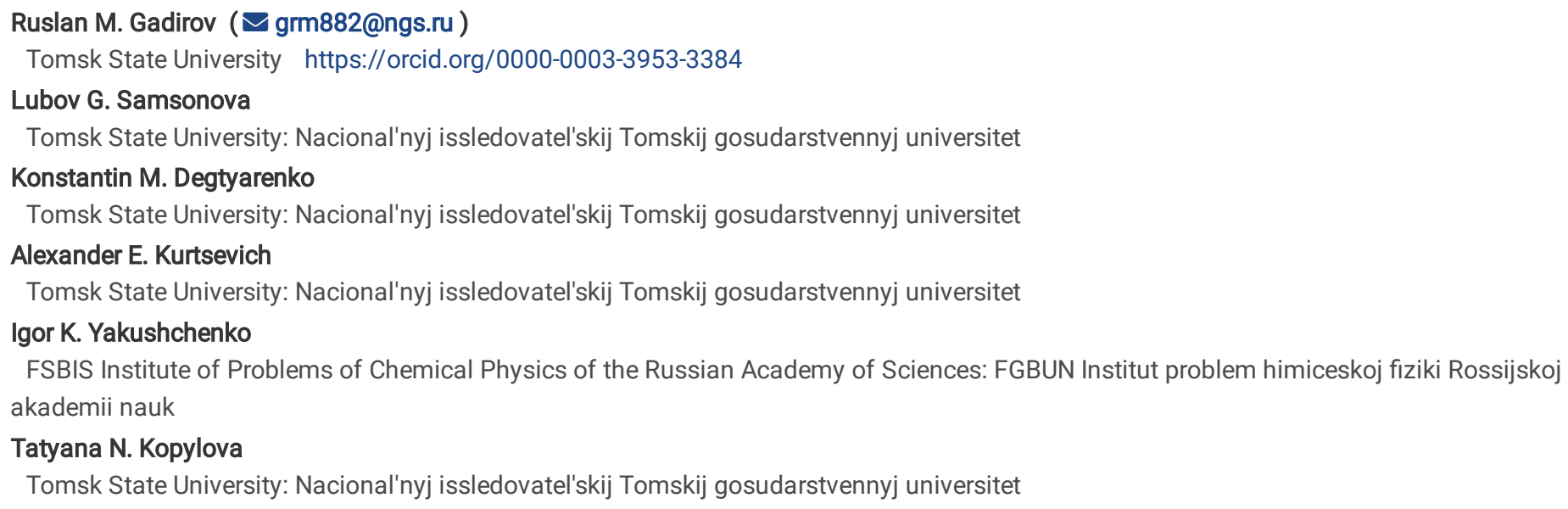

Keywords: dibenzothiophene sulfone, phenantridine, dipole moment, fluorescence, delayed fluorescence, phosphorescence, electroluminescence, organic light-emitting diodes

Posted Date: February 19th, 2021

DOI: https://doi.org/10.21203/rs.3.rs-210952/v1

License: (c) (i) This work is licensed under a Creative Commons Attribution 4.0 International License. Read Full License 


\section{Abstract}

Spectral characteristics and luminescence under the photo- and electro-excitation of substituted dibenzthiophene sulfone and phenanthridine were studied in this paper. Diphenylamines are substituents introduced in the 2nd and 7th positions (linear configuration) or the 3rd and 6th positions (angular configuration) of dibenzthiophene sulfone or phenanthridine. All molecules show delayed fluorescence, both in solutions and films produced by thermal vacuum deposition. The value of the energy gap between the $S_{1}$ and $T_{1}$ states has been estimated and is shown to depend not only on the spatial arrangement of the fragments among themselves (linear or angular), but also on the nature of the substituent in diphenylamine. The highest electroluminescence brightness was found for the molecules, in which triplet levels are involved, both through the process of triplet-triplet annihilation and through thermally activated delayed fluorescence.

\section{Introduction}

Organic molecules, which emit light in the field of applied voltage, have been widely used to create organic light-emitting diodes (OLEDs) in recent decades. Currently, screens of mobile phones, tablets, television sets, and on-board car computers are manufactured using OLEDs. Along with the obvious advantages of organic LEDs, such as high contrast and brightness, the ability to create devices on the flexible base surface and relative ease of manufacturing, there are a number of problems that scientists continue to work on. One of them is low internal quantum efficiency. It is related to the formation characteristics of light-emitting centres in the OLED structure during electroexcitation. According to quantum statistics, $75 \%$ of the created excitons are in the triplet state and only $25 \%$ are in the singlet []. It is known that the triplet state of most organic molecules is nonradiative, especially at room temperature. The exception is metal-organic complexes based on precious metals, such as iridium []. The OLEDs using such materials are highly effective, but quite expensive.

In 2012, in order to increase the efficiency of OLED structures, Adachi [ proposed the use of molecules with a high probability of reverse intersystem crossing (RISC) from the $\mathrm{T}_{1}$ state to the $\mathrm{S}_{1}$ state. In this case, a large proportion of the triplet excitons emit light in the form of thermally activated delayed fluorescence (TADF). To date, a large set of small molecules, dendrimers and polymers with TADF luminescence has been proposed for OLED [,]. The key factor of RISC is the value of the energy gap between the $S_{1}$ and $T_{1}$ states. The smaller it is, the more likely the process of reverse intersystem crossing. The TADF mechanism allows reaching the theoretical limit of the internal quantum efficiency of $100 \%$. It's known [ that a small energy gap between the $S_{1}$ and $T_{1}$ states is achieved when the donor and acceptor fragments are orthogonal in the TADF emitter. Such geometry leads to a small value of the exchange integral, since the HOMO and LUMO orbitals do not spatially overlap, but the oscillator strength of the emitting charge-transfer state also decreases strongly.

Another mechanism for increasing the efficiency of OLED by overcoming the spin-statistical limit of fluorescent molecules is triplet-triplet annihilation (TTA) []. On the one hand TTA is less efficient because the maximum internal efficiency at such mechanism can reach only 62.5\% [11]. On the other hand, the TTA mechanism does not require an orthogonal arrangement of donor and acceptor fragments, as in the case of TADF. Thus, the quantum yields of fluorescence of such TTA emitters are usually higher.

In this paper, the photophysical and electroluminescent properties of molecules with the possible RISC and TTA processes have been investigated (Fig. 1). Previously [ the data of quantum chemical calculations of the rate constants of photophysical processes, such as radiation decay, internal conversion, forward and reverse intersystem crossings, and energy gaps between the $S_{1}$ and $T_{1}$ states were described for these compounds. The compounds have been experimentally investigated here and their correspondence with the theoretical data has been established [12].

\section{Experimental Details}

The spectral properties of the compounds were studied in solutions of ethanol, chloroform and films obtained by thermal vacuum deposition on quartz base surfaces (TVD). Absorption spectra in the UV-visible region were recorded using a CM2203 spectrophotometer (SOLAR, Belarus), fluorescence spectra, phosphorescence and phosphorescence decay times were recorded on a Cary Eclipse spectrofluorometer. The quantum yield of fluorescence in ethanol and chloroform solutions was estimated by the absolute method according to technique [[i]].

The phosphorescence of compounds was studied in ethanol and TVD films at a decreasing temperature from room temperature to 77K in steps of 30 degrees and a delay in the monitoring time of $200 \mu$ s after the lamp flash. Preliminary deoxygenation of solutions was not carried out. The energy gap between the $S_{1}$ and $T_{1}$ states was estimated from the position of the maxima of the fluorescence and phosphorescence bands at temperatures of $77 \mathrm{~K}$.

The phosphorescence lifetimes were estimated by approximating the radiation attenuation curve with one or more exponential functions: .

$$
\mathrm{I}(\mathrm{t})=A_{1} \exp \left(-t / \tau_{1}\right)+A_{2} \exp \left(-t / \tau_{2}\right)+\ldots A_{n} \exp \left(-t / \tau_{n}\right)
$$

To create the OLED structures, glass base surfaces coated with a transparent layer of a mixture of indium and tin oxides (ITO) with a resistance of $12 \mathrm{Ohms} / \square$ were used. 
Anode tracks were made on the base surfaces by laser engraving. Preliminary preparation of the base surfaces was carried out according to the established method: thorough cleaning in organic solvents followed by etching in oxygen plasma.

The depositing of layers onto the prepared base surface was carried out in a glove box in a dry nitrogen atmosphere. Thermal vacuum deposition (TVD) was performed on an AUTO 306 unit by BOC EDWARDS using shadow masks at a residual pressure of $\sim 10^{-5} \mathrm{mbar}$ and a deposition rate of $0.2 \mathrm{~nm} / \mathrm{s}$ for organic layers and $2 \mathrm{~nm} / \mathrm{s}$ for metals. The size of the active area was $4 \times 4 \mathrm{~mm}^{2}$.

The layers of organic substances and metals of the cathode were created without depressurization of the chamber. The speed and thickness of the deposited layer were monitored by SQM 160 quartz detector.

The volt-ampere, volt-brightness and spectral characteristics of the obtained OLED structures were studied using a measuring complex consisting of a Keithley 237 voltage analyzer source and an AvaSpec-ULS-2048x64 fibre spectrometer.

Electroluminescent properties were studied in the composition: ITO/ PEDOT:PSS /NPD/EML/BCP/LiF/Al.

PEDOT:PSS - 2.8 wt. \% aqueous solution of Poly(3,4-ethyldioxythiophene): Poly(styrenesulfonate) (Aldrich) was deposited to ITO by centrifugation at a speed of $4000 \mathrm{r} / \mathrm{min}$ for 30 seconds and dried at a temperature of $120^{\circ} \mathrm{C}$. This substance not only used to smooth the surface of the anode but also serves as an emitter of holes. The thickness of the PEDOT:PSS films was $40 \mathrm{~nm}$.

NPD - N4,N4'-di(naphthalen-1-yl)-N4,N4'-diphenylbiphenyl-4,4'-diamine (Aldrich) was deposited using TVD method. The layer thickness was 40 nm. This compound serves as a transporter of holes in sandwich structures.

EML is a radiating layer with a thickness of $50 \mathrm{~nm}$.

BCP -2,9-dimethyl-4,7-diphenylbenzo[h]quinoline (Aldrich), $20 \mathrm{~nm}$ thick, was used as electron transport material and for blocking the holes.

The studied compounds shown in Fig. 1 were synthesized according to the previously described procedure [7].

\section{Results And Discussion}

L1 and A1 compounds have the same fragments in the molecular structure: the electron-acceptor dibenzothiophene-S,S-dioxide and two electrondonating diphenylamine fragments connected to the central fragment via meta- and para-positions with respect to the $\mathrm{SO}_{2} \mathrm{group}$. In the $\mathbf{A 2}$ compound, in comparison with A1, the tretbutyl groups are replaced by methoxy groups. Methoxy substitution is followed by a small ( $6 \mathrm{~nm})$ blue shift of the main absorption bands. In the L2, the central acceptor fragment consists of phenylphenanthridine.

Since the mutual arrangement of the diphenylamine fragments and the central fragment leads to a noticeable change in the spectral properties, we shall conditionally designate L1 and L2 as compounds of linear structure, and A1 and A2 as those of angular structure. Thus, linear compounds have a band in the absorption spectra in the region of $\sim 440 \mathrm{~nm}$ (Fig. 2), while angular molecules do not have one. At the same time, an abnormally large Stokes shift of $7000-9000 \mathrm{~cm}^{-1}$ is observed in all the studied media for the A1 and A2 compounds of angular structure (Table 1). This fact is clarified by quantum chemical calculations [12]. The long-wave absorption band of these compounds appears to be formed by the $\mathrm{S}_{0}-\mathrm{S}_{3}$ transition, whereas the $\mathrm{S}_{0}-\mathrm{S}_{1}$ transition has very low oscillator strength $(\sim 0.08)$ due to its charge-transfer nature and it is located on the red wing of the absorption band.

It should be noted that all compounds are characterized by a weak solvatochromic dependence, i.e. the change of the ambient medium (ethanol, chloroform and TVD film) has almost no effect on the position of the bands in the absorption spectra (Table 1, 2 and Fig. S1). 
Table 1

Spectral-luminescent properties of compounds in $\mathrm{CHCl}_{3}$, ethanol and TVD-films.

\begin{tabular}{|c|c|c|c|c|c|c|c|c|c|c|c|c|}
\hline Compound & L1 & & & A1 & & & L2 & & & $\mathrm{A} 2$ & & \\
\hline Media & $\mathrm{CHCl}_{3}$ & Ethanol & TVD & $\mathrm{CHCl}_{3}$ & Ethanol & TVD & $\mathrm{CHCl}_{3}$ & Ethanol & TVD & $\mathrm{CHCl}_{3}$ & Ethanol & TVD \\
\hline \multirow[t]{2}{*}{$\lambda_{\mathrm{abs}}, \mathrm{nm}$} & 382 & 376 & 380 & \multirow[t]{2}{*}{362} & \multirow[t]{2}{*}{360} & \multirow[t]{2}{*}{360} & 377 & 370 & 370 & \multirow[t]{2}{*}{358} & \multirow[t]{2}{*}{350} & \multirow[t]{2}{*}{356} \\
\hline & 440 & 432 & 432 & & & & 433 & 440 & 440 & & & \\
\hline $\begin{array}{l}\lambda_{\mathrm{PF}}^{296}\left(\lambda_{\mathrm{PF}}{ }^{77}\right)^{*}, \\
\mathrm{~nm}\end{array}$ & 525 & $\begin{array}{l}538 \\
(483 / 513)\end{array}$ & $\begin{array}{l}517 \\
(518 / 539)\end{array}$ & 512 & $\begin{array}{l}548 \\
(467)\end{array}$ & $\begin{array}{l}485 \\
(488)\end{array}$ & 518 & $\begin{array}{l}526 \\
(504)\end{array}$ & $\begin{array}{l}502 \\
(501)\end{array}$ & 553 & $\begin{array}{l}605 \\
(501)\end{array}$ & $\begin{array}{l}535 \\
(530)\end{array}$ \\
\hline$\Delta \mathrm{v}_{\text {Stokes }}, \mathrm{cm}^{-1}$ & 3680 & 4560 & 3810 & 8090 & 9530 & 7160 & 3790 & 3720 & 2810 & 10330 & 12040 & 9040 \\
\hline PLQY $296 K$ & 0.94 & 0.46 & 0.2 & 0.2 & 0.2 & 0.06 & 0.2 & 0.15 & 0.03 & 0.4 & 0.02 & 0.13 \\
\hline $\begin{array}{l}\lambda_{\mathrm{DL}}{ }^{296}\left(\lambda_{\mathrm{DL}}{ }^{77}\right) \\
\mathrm{nm}\end{array}$ & - & 511 & 527 (539) & - & 465 & 488 & - & 530 & - & - & 605 & - \\
\hline$\lambda_{\text {Phos }}{ }^{77}, \mathrm{~nm}$ & - & 596 & $632 / 687$ & - & 523 & 535 & - & 566 & 570 & - & $534 / 565$ & $560 / 591$ \\
\hline$\tau_{\text {phos } 1} / \tau_{\text {phos } 2}{ }^{\star *}$ & - & $2.4 /$ & - & - & 200 & $16.8 /$ & - & 770 & $12.9 /$ & - & 113 & $16.6 /$ \\
\hline $\mathrm{ms}$ & & 11.5 & & & & 68.9 & & & 158 & & & 66.2 \\
\hline$\Delta\left(\mathrm{E}_{\mathrm{S} 1}-\mathrm{E}_{\mathrm{T} 1}\right), \mathrm{ev}$ & & 0.49 & 0.43 & & 0.28 & 0.22 & & 0.27 & 0.30 & & 0.15 & 0.13 \\
\hline $\begin{array}{l}\Delta\left(E_{S 1}-E_{T 1}\right)^{\text {calc }}, \\
\text { ev [12] }\end{array}$ & 0.34 & & & 0.13 & & & - & & & 0.13 & & \\
\hline * the position o & the mos & intense vibrc & ic transition & s indicat & d via a sla & & & & & & & \\
\hline ** phosphoresc & ce lifet & nes by two-e & oonential de & & & & & & & & & \\
\hline
\end{tabular}

The fluorescence spectra are more sensitive to the polarity of the solvent. Thus, the $\mathbf{A} 1$ in hexane emits with a maximum at 442 nm, and in acetonitrile, it emits with a maximum at $558 \mathrm{~nm}$. This means that changing non-polar to strongly polar solvent, the fluorescence band shifts to the red region by $116 \mathrm{~nm}$. The second $\mathbf{A} 2$ compound of angular structure undergoes an even greater shift - $174 \mathrm{~nm}$. The red shift of linear compounds is smaller (74 and $40 \mathrm{~nm}$ for the L1 and L2 respectively). The dependences of the maxima position of the absorption and fluorescence bands on the solvent are shown in Table 2 and Fig. S1. The higher the polarity of the solvent is, the broader the fluorescence bands are. For angular molecules, the bands in acetonitrile are $50-60 \%$ wider than in hexane. For linear molecules, this value is $15-17 \%$. A noticeable difference in the half-widths of the fluorescence bands of linear and angular molecules in polar solvents indicates a greater variety of geometric conformers of the latter in the electron-excited state. The red shift of radiation with increasing polarity of the solvent indicates a strong redistribution of the electron density in the molecule in the $\mathrm{S}_{1}$ electron-excited state. An increase in intermolecular interactions with the solvent in the excited state can be expected to be due to a significant increase in the dipole moment in the $\mathrm{S}_{1}$ state.

From the previously obtained data [12], it is known that the $S_{0} \rightarrow S_{1}$ transitions are of low intensity and they are located on the wing of more intense high-energy transitions in the $\mathbf{A} 1$ and $\mathbf{A} 2$ angular molecules. Therefore, the dipole moments from solvate models cannot be determined for these molecules. However, in the L1 and L2 linear molecules the $S_{0} \rightarrow S_{1}$ transitions have sufficiently high intensities, their position can be determined from the absorption spectra and the dipole moments in the $S_{0}$ and $S_{1}$ states can be calculated for them.

The Bilot-Kawski model was used to estimate the dipole moments of molecules in the $\mathrm{S}_{0}$ and $\mathrm{S}_{1}$ states []. In contrast to the Lippert-Mataga model, this model gives a more accurate agreement with the experiment [14]. The Supplementary Material provides a method for calculating dipole moments using the Bilot-Kawski model and shows the values of the dipole moments of the L1 and L2 molecules (Table S1). The dipole moments of both molecules have been found to be small in the ground state and they amount to 0.61 and 1.05 Debye for the L1 and L2 respectively. During transition to the $S_{1}$ state, the dipole moments increase (6.96 and 6.08 Debye for the L1 and L2 respectively). This suggests that even in the molecules with an almost linear arrangement of donor substituents, there is a significant redistribution of the electron density. Therefore, even greater values of the dipole moments themselves should be expected both for the molecules with the angular arrangement of substituents and changes during transition to the excited state. These results confirm the data of quantum chemical calculations [12] and the assumption about the charge-transfer nature of the $\mathrm{S}_{0} \rightarrow \mathrm{S}_{1}$ transition in such molecules.

It is characteristic that the position of the fluorescence maxima of all compounds in TVD films is close to those in weakly polar solvents (toluene and chloroform). This suggests that in TVD films, with the molecules in the sample packed more closely than in low-concentration solutions, the 
compounds do not enter into specific intermolecular interactions and retain their individual properties. The quantum yield of fluorescence in TVD films has also been found to decrease significantly compared to chloroform solutions (Table 1). These compounds are therefore characterized by concentration quenching since the fluorescence intensity has not changed in the argon atmosphere.

Table 2

Maxima of absorption and fluorescence wavelengths of compounds in various solvents

\begin{tabular}{|c|c|c|c|c|c|c|c|c|c|c|}
\hline \multirow[t]{2}{*}{ Solvent } & \multirow[t]{2}{*}{$\boldsymbol{\varepsilon}$} & \multirow[t]{2}{*}{$\mathbf{n}$} & \multicolumn{2}{|l|}{ L1 } & \multicolumn{2}{|l|}{$\mathrm{A} 1$} & \multicolumn{2}{|l|}{ L2 } & \multicolumn{2}{|l|}{ A2 } \\
\hline & & & $\lambda_{\text {abs, }}, \mathrm{nm}$ & $\lambda_{\mathrm{fl}}, \mathrm{nm}$ & $\lambda_{\mathrm{abs}}{ }^{*}, \mathrm{~nm}$ & $\lambda_{\mathrm{fl}}, \mathrm{nm}$ & $\lambda_{\mathrm{abs}}, \mathrm{nm}$ & $\lambda_{\mathrm{fl}}, \mathrm{nm}$ & $\lambda_{\mathrm{abs}}{ }^{*}, \mathrm{~nm}$ & $\lambda_{\mathrm{fl}}, \mathrm{nm}$ \\
\hline n-Hexane & 1.9 & 1.375 & 423 & 463 & 355 & 442 & 420 & 480 & 350 & 466 \\
\hline Toluene & 2.38 & 1.494 & 433 & 490 & 367 & 478 & 431 & 492 & 355 & 516 \\
\hline Chloroform & 4.78 & 1.443 & 440 & 525 & 362 & 512 & 433 & 518 & 358 & 553 \\
\hline Chlorobenzene & 5.63 & 1.525 & 432 & 508 & 363 & 507 & 431 & 505 & 357 & 549 \\
\hline Ethyl acetate & 6.00 & 1.370 & 425 & 500 & 359 & 509 & 422 & 499 & 353 & 549 \\
\hline Tetrahydrofuran & 7.22 & 1.404 & 424 & 498 & 360 & 509 & 425 & 498 & 354 & 551 \\
\hline Acetone & 20.8 & 1.356 & 425 & 516 & 358 & 546 & 429 & 513 & 352 & 609 \\
\hline Ethanol & 25.3 & 1.361 & 432 & 538 & 360 & 548 & 440 & 526 & 350 & 605 \\
\hline Dimethylformamide & 36.7 & 1.428 & 428 & 536 & 358 & 550 & 428 & 520 & 352 & 624 \\
\hline Acetonitrile & 38.8 & 1.342 & 430 & 537 & 357 & 558 & 422 & 526 & 351 & 640 \\
\hline Dimethyl sulfoxide & 48.9 & 1.476 & 434 & 540 & 361 & 563 & 428 & 530 & 354 & 632 \\
\hline
\end{tabular}

Monitoring of luminescence spectra, when decreasing the temperature in steps of $30^{\circ}$ from room temperature to $77 \mathrm{~K}$, was carried out both in the Prompt Fluorescence mode and in the Delayed Luminescence mode, i.e. $200 \mu$ s after the flash of the excitation lamp with a registration duration of $5 \mathrm{~ms}$. In the Delayed Luminescence mode, both delayed fluorescence and phosphorescence were recorded. The delayed fluorescence was observed at room temperature and disappeared (or almost disappeared) as the temperature decreased. The phosphorescence in the form of a new band of radiation appeared in the red region of the spectrum and, as a rule, its intensity increased strongly with a decrease in temperature. Such studies were carried out both in ethanol solution and in TVD films.

Absorption and fluorescence spectra in ethanol. A decrease in the temperature of ethanol solutions of compounds leads to a bathochromic shift of the absorption bands (a change in the absorption position was recorded in the fluorescence excitation spectra. Fig. S3). Similar effects were observed in the following works [,]. It is known that when the temperature decreases, both macroscopic parameters increase - the refractive index of the medium $\left(n_{D}\right)$ and the dielectric constant $\left(\varepsilon_{D}\right)$ which affect the shape and position of the absorption and fluorescence bands. As mentioned above, the absorption spectra of the compounds practically did not shift (Fig. S1) when changing the solvent from $\varepsilon_{D}=1.9$ (hexane) to $\varepsilon_{D}=48.9$ (dimethylsulfoxide). Consequently, this parameter did not significantly affect the red shift of the absorption spectra as the temperature decreased.

The increase of $n_{D}$ boosts the power of dispersion interactions, which are stronger in the excited state than in the ground state due to higher polarizability. For this reason, the energy level of the Frank-Condon excited state of the $S_{1 F C}$ decreases more strongly than the $S_{0}$ state. As a consequence, the bathochromic shift of the dye absorption bands during the cooling stage can be explained by increasing $n_{D}, E\left(S_{0} \rightarrow S_{1 F C}\right)^{77}$ $<\mathrm{E}\left(\mathrm{S}_{0} \rightarrow \mathrm{S}_{1 \mathrm{FC}}\right)^{293}$, where $\mathrm{E}$ is the electron transition energy.

Figure 3 shows the fluorescence spectra of compounds in ethanol solution at a decreasing temperature from room temperature to $77 \mathrm{~K}$. With a decrease in temperature, all ethanol solutions of the studied compounds are characterized by a hypsochromic shift of the fluorescence band. This behaviour is associated with a change in the solvent relaxation time which increases with an increase in the number of hydrogen bonds formed between alcohol molecules [] as the temperature decreases up to the solid-phase state at $\mathrm{T} \approx 158 \mathrm{~K}$. With an increase in the number of hydrogen bonds in the solvate shell, resolvation of the dissolved molecules slows down during the lifetime of the excited state, which is reflected in the blue shift of the fluorescence bands and a decrease in their half-width by an average of $25 \%$.

Interestingly, the fluorescence intensity of molecules with angular substituents $(\mathbf{A} 1, \mathbf{A} 2)$ passes through the maximum as the temperature decreases (Fig. 3, S13, S15). The intensity increases approximately up to the glass transition temperature (the quantum yield of fluorescence increases), but a further decrease in the temperature to $77 \mathrm{~K}$ is followed by a decrease in the fluorescence intensity. The reason for this phenomenon is still unclear. 
The fluorescence spectra in TVD films. The fluorescence behaviour of compounds in TVD films is very different from that in ethanol solution. When the temperature decreases, the position of the bands in the spectra practically does not change (Fig. S16-S19), but the radiation intensity of the L1, A1 and L2 compounds at temperatures below 150K drops sharply (Fig. S8a-S11a). In the A2 compound in the temperature range from 296 to $77 \mathrm{~K}$, the intensity changes are less significant (within 20\%). The reason for the different behaviour of fluorescence of compounds in TVD films at a decreasing temperature is still difficult to comment on.

Delayed fluorescence and phosphorescence. The radiation spectra recorded with a delay of $200 \mu \mathrm{s}$ after the lamp flash at room temperature in ethanol solutions coincide in position and shape with the prompt fluorescence spectra (Fig. S4-S7). The coincidence of the radiation bands provides grounds to believe that the recorded radiation belongs to delayed fluorescence.

The delayed emission at room temperature of TVD films of compounds, except the L1, differs from prompt fluorescence (Fig. S8-S11). The maxima of the delayed luminescence bands of the A1, L2 and A2 films are shifted to the red region with respect to fluorescence (Fig. 4).

Weak delayed luminescence is observed at room temperature for the L1 compound. Unlike other compounds, delayed fluorescence of L1 is observed over the entire temperature range (Fig. S8). However, a phosphorescence band appears in the long-wave region at temperatures below 150K. Delayed fluorescence of L1 may be caused by the process of the triplet-triplet annihilation (TTA) as was observed for this molecule in [7]. The long-lived radiation for all other compounds lies in the longer wavelength region relative to fluorescence already at room temperature (Fig. 4) and can be attributed to mix of delayed fluorescence and phosphorescence. With a decrease in temperature, the intensity of phosphorescence increases significantly. The greatest increase in intensity (180 times) is observed for the A1 film. A clear separation according to the position in the spectrum into fluorescence and phosphorescence at low temperatures for the L1, L2 and A1 allows for estimating the energy gap $\Delta_{S T}$ between the $S_{1}$ and $T_{1}$ states. Delayed luminescence of the $\mathbf{A} 2$ compound at room temperature lies in almost the same region as fluorescence, but the radiation band is broadened on the red wing side. With a decreasing temperature, the intensity of this band increases and a narrowing appears on the side of the blue wing (Fig. S11).

Table 1 shows $\Delta_{S T}$ values for both ethanol solutions and films. It can be seen that these values are close to each other in solutions and films and they correlate well with the data of quantum chemical calculations [12]. The lowest value of $0.13-0.15 \mathrm{eV}$ is observed for the $\mathbf{A} 2$ compound. Such a small value of $\Delta_{\mathrm{ST}}$ leads to a significant overlap of the fluorescence and phosphorescence bands, increases the rate constant of reverse intersystem crossing and the intensity of thermally activated delayed fluorescence. In addition, it is known [12] that the presence of auxiliary methoxyl groups in the donor fragments of the $\mathbf{A} \mathbf{2}$ compound leads to an increase in the matrix element of the spin-orbit interaction by an order compared to $\mathbf{A} 1$, which also increases the yield of TADF. Thus, we can expect that the electroluminescence intensity of the OLED structure with the A2 should be sufficiently high due to the borrowing of triplet excitons by the mechanism of thermally activated delayed fluorescence.

The phosphorescence kinetics can be described by a two-exponential decay in TVD films (Table 1). The molecules might be in more than one molecular conformation with a denser packing of molecules in the process of thermal vacuum deposition in the film.

\section{Electroluminescent Properties}

Figures 5, S20-S23 and Table 3 show the electroluminescent characteristics of nondoped OLEDs with the following structure: ITO/PEDOT:PSS/NPD/EML/BCP/LiF/Al, where EML is an emitting layer based on the studied compounds. The electroluminescence spectra of all compounds in OLED structures practically coincide with the photoluminescence spectra in TVD films (Fig. S25). It can be concluded that no new emitting forms (for example, exciplexes) are formed in OLED structures, and electroluminescence occurs from the $\mathrm{S}_{1}$ state. The highest quantum efficiency $\mathrm{EQE}_{\max }$ equal to $1.53 \%$ is achieved for the OLED structure based on L1 (Table 3), while the angular form A2 exhibits an efficiency of only $0.79 \%$. Such low values of the external quantum efficiency are due to the low value of the fluorescence quantum yields of compounds in TVD-films. It is known that the external quantum efficiency EQE $=\eta_{\text {out }} \times \gamma_{\mathrm{eh}} \times \beta_{\mathrm{ST} \times} \varphi_{\mathrm{fl}}$ ] where $\eta_{\text {out }}$ is the coefficient of light output from the OLED structure, $\gamma_{\mathrm{eh}}$ is a factor showing the efficiency of electron-hole recombination (for well balanced structures is close to $100 \%$ ), $\beta_{\mathrm{ST}}$ is the spin statistical limit (it results on the ratio of the probability of formation of singlet and triplet excitons at $1: 3$ and constitutes generally $25 \%$ for singlet emitters), $\varphi_{\mathrm{fl}}-$ fluorescence quantum yield. Assuming $Y_{e h}=1$ and $\eta_{\text {out }}=0.2$ for glass substrate $[$ and taking into account the fluorescence quantum yields of compounds in TVD films (Table 1), we can estimate $\beta_{S T}$ (Table 3). It can be seen that the $25 \%$ barrier is overcome for L1 and A2. This suggests that electroluminescence occurs with the participation of triplet states. Since $\Delta_{\mathrm{ST}}$ for compound $\mathbf{A} 2$ is only $0.13 \mathrm{eV}$ (Table 1 ), it can be argued that in the OLED structure based on this compound, the $\beta_{\mathrm{ST}}$ barrier is overcome by the TADF mechanism. The energy gap $\Delta_{\mathrm{ST}}$ of compound L1 is 0.43 eV, so the TADF process in this structure improbable. However, it is known [7] that triplet-triplet annihilation (TTA) takes place for this compound. It also allows the activation of triplet levels, thereby contributing to the emission of OLED structures. 
Table 3

Electrolumence characteristics [emission maxima $\boldsymbol{\lambda}_{\mathbf{e}}$, turn-on voltage $\mathbf{V}_{\text {on }}$ (at brightness

$\left.1 \mathrm{~cd} / \mathrm{m}^{2}\right)$, maximal brightness $B_{\max }$ at voltage $(V)$, current efficiency $\varphi(1000)$ and external quantum efficiency $\mathrm{EQE}_{1000}$ (at brightness of $1000 \mathrm{~cd} / \mathrm{m}^{2}$ ), $\mathrm{EQE}_{\max }$ and spin-statistical factor $\left.\boldsymbol{\beta}_{\mathrm{ST}}\right]$ of OLED structures.

\begin{tabular}{|lllllll|}
\hline Compound & $\lambda_{\mathrm{el}}, \mathrm{nm}$ & $\mathrm{U}_{\mathrm{on}}, \mathrm{V}$ & $\mathrm{B}_{\mathrm{max}}, \mathrm{cd} / \mathrm{m}^{2}(\mathrm{~V})$ & $\varphi(1000), \mathrm{cd} / \mathrm{A}$ & $\mathrm{EQE}_{\max }, \% /$ & $\beta_{\mathrm{ST}}, \%$ \\
$\mathrm{EQE}_{1000}, \%$ & \\
\hline L1 & 515 & 3.9 & $4500(12)$ & 4.50 & $1.53 / 1.47$ & 38.3 \\
\hline A1 & 490 & 5.2 & $1510(15)$ & 0.58 & $0.30 / 0.28$ & 24.6 \\
\hline L2 & 492 & 6.0 & $615(14)$ & $0.10^{*}$ & $0.06 / 0.04^{*}$ & 9.2 \\
\hline A2 & 527 & 4.2 & $2330(12)$ & 2.34 & $0.79 / 0.78$ & 30.4 \\
\hline * The values are given at a maximum achieved brightness of $615 \mathrm{~cd} / \mathrm{m}^{2}$. & \\
\hline
\end{tabular}

\section{Conclusion}

It was shown that an angular arrangement of donor and acceptor fragments in the $\mathbf{A} 1$ molecule leads to a decrease in the quantum yield of fluorescence compared to the linear arrangement of the fragments in L1. In addition TTA process observed for L1 increases the efficiency of OLED structure.

Replacement of the tretbutyl groups of the A1 with the methoxy groups of the $\mathbf{A} 2$ leads to a red shift of the fluorescence bands and reduction in the energy gap between the $S_{1}$ and $T_{1}$ states, which facilitates reverse intersystem crossing from the $T_{1}$ to $S_{1}$ and promotes electroluminescence due to TADF.

\section{Declarations}

\section{Availability of data and materials}

The datasets supporting the conclusions of this article are included within the article and its additional files.

\section{Competing Interest}

The authors declare no competing financial interest.

\section{Funding}

This work was supported by The Tomsk State University competitiveness improvement program under grant No. 8.1.38.2020.

\section{Authors' contributions}

R.M. Gadirov Low-temperature measurements, calculating dipole moments, writing an article

L.G. Samsonova Performing low-temperature and solvatochromic studies, processing spectral data, writing an article

K.M. Degtyarenko Creation of OLEDs, measurement of their current-voltage, brightness-voltage and spectral characteristics

A.E. Kurtsevich Spectral data processing, creating charts

I.K. Yakushchenko Synthesis of the studied compounds

T.N. Kopylova General project management, article correction

\section{Ethical approval}

Not applicable

\section{Code availability}

Not applicable 


\section{References}

1. A. Baldo, D.F. O'brien, M.E. Thompson, S.R. Forrest Excitonic singlet-triplet ratio in a semiconducting organic thin film. Phys. Rev. B 60.20 (1999) 14422 doi:10.1103/PhysRevB.60.14422.

2. Minaev, G. Baryshnikov, H. Agren Principles of phosphorescent organic light emitting devices // Phys. Chem. Chem. Phys. 16 (2014) $1719-1758$ doi:10.1039/С3CP53806K.

3. A. Baldo, D.F. O'Brien, Y. You, A. Shoustikov, S. Sibley, M.E. Thompson, S.R. Forrest Highly efficient phosphorescent emission from organic electroluminescent devices // Nature 395.6698 (1998) 151-154. doi:10.1038/nature11687.

4. J. Su, E. Gonmori, H. Sasabe, J. Kido Highly efficient organic blue-and white-light-emitting devices having a carrier-and exciton-confining structure for reduced efficiency roll-off // Adv. Mater. 20.21 (2008) 4189-4194 doi:10.1002/adma.200801375.

5. Uoyama, K. Goushi, K. Shizu, H. Nomura, C. Adachi Highly efficient organic light-emitting diodes from delayed fluorescence// Nature 492.13 (2012) 234-240 doi:10.1038/nature11687.

6. Hu, L. Yao, B. Yang, Y. Ma Reverse intersystem crossing from upper triplet levels to excited singlet: a 'hot excition' path for organic light-emitting diodes // Phil. Trans. R. Soc. A. 373.2044 (2015) 20140318. doi:10.1098/rsta.2014.0318.

7. B. Dias, K.N. Bourdakos, V. Jankus, K.C. Moss, K.T. Kamtekar, V. Bhalla, J. Santos, M.R. Bryce, A.P. Monkman Triplet harvesting with $100 \%$ efficiency by way of thermally activated delayed fluorescence in charge transfer OLED emitters, Adv. Mater. 25.27 (2013) 3707-3714 doi:10.1002/adma.201300753.

8. Liu, C. Li, Z. Ren, S. Yan, M.R. Bryce All-organic thermally activated delayed fluorescence materials for organic light-emitting diodes // Nat. Rev. Mater. 3.4 (2018) 18020 doi:10.1038/natrevmats.2018.20.

9. Yang, Z. Mao, Z. Xie, Y. Zhang, S. Liu, J. Zhao, J. Xu, Z. Chi, M.P. Aldred. Recent advances in organic thermally activated delayed fluorescence materials // Chem. Soc. Rev. 46.3 (2017) 915-1016. doi:10.1039/C6CS00368K.

10. Y. Kondakov Characterization of triplet-triplet annihilation in organic light-emitting diodes based on anthracene derivatives //J. Appl. Phys. 102.11 ( 2007) 114504. doi:10.1063/1.2818362

11. D.Y.Kondakov, T.D. Pawlik, T.K. Hatwar, J.P. Spindler Triplet annihilation exceeding spin statistical limit in highly efficient fluorescent organic light-emitting diodes // J. Appl. 106 (2009) 124510. doi:10.1063/1.3273407.

12. M. Gadirov, R.R. Valiev, L.G. Samsonova, K.M. Degtyarenko, N.V. Izmailova, A.V. Odod, S.S. Krasnikova, I.K. Yakuschenko, T.N. Kopylova Thermally activated delayed fluorescence in dibenzothiophene sulfone derivatives: theory and experiment // Chem. Phys. Let. 717 (2019) 53-58 doi:10.1016/j.cplett.2019.01.014.

13. C. de Mello, H.F. Wittmann, R.H. Friend An improved experimental determination of external photoluminescence quantum efficiency // Adv. Mater. 9 (1997) 230-232 doi:10.1002/adma.19970090308.

14. Kawski On the estimation of excited-state dipole moments from solvatochromic shifts of absorption and fluorescence spectra // Z. Naturforsch. 57 (2002) 255-262 doi:10.1515/zna-2002-0509.

15. S. Raikar, C.G. Renuka, Y.F. Nadaf, B.G. Mulimani, A.M. Karguppikar, M.K. Soudagar Solvent effects on the absorption and fluorescence spectra of coumarins 6 and 7 molecules: Determination of ground and excited state dipole moment. // Spectrochim. Acta A. 65.3-4 (2006) 673-677 doi:10.1016/j.saa.2005.12.028.

16. Lippert, W. Lüder, F. Moll Polarisations-und Relaxations-Effekte in der Temperaturabhängigkeit von Absorptions-und Fluoreszenzspektren aromatischer Verbindungen in polaren Lösungsmitteln // Spectrochim. Acta. 15 (1959) 858-869 doi:10.1016/S0371-1951(59)80383-0.

17. A. Ishchenko, A.V. Kulinich, S.L. Bondarev, V.N. Knyukshto, A.A. Turban Thermochromism and thermofluorochromism of merocyanines with a positive solvatochromism // Optics and spectroscopy, 101.1 (2006) 90-97 doi:10.1134/S0030400X06070162.

18. M. Dinastiya, E.V. Verbitskiy, R.M. Gadirov, L.G. Samsonova, K.M. Degtyarenko, D.V. Grigoryev, A.E. Kurtcevich, T.A. Solodova, E.N. Tel'minov, G.L. Rusinov, O.N. Chupakhin, V.N. Charushin Investigation of 4,6-di(hetero)aryl-substituted pyrimidines as emitters for non-doped OLED and laser dyes // J. Photochem. Photobiol. A. 408 (2021) 113089. doi:10.1016/j.jphotochem.2020.113089

19. G. Su, J.D. Simon Solvation dynamics in ethanol // J. Phys. Chem. 91.11 (1987) 2693-2696 doi:10.1021/j100295a008.

20. Maroncelli The dynamics of solvation in polar liquids // J. Mol. Liq. 57 (1993) 1-37 doi:10.1016/0167-7322(93)80045-W.

21. Liu, Z. Li, T. Hu, X. Wei, R. Wang, X. Hu Experimental Evidence for “Hot Exciton” Thermally Activated Delayed Fluorescence Emitters // Adv. Optical Mater. 7 (2019) 1801190 doi:10.1002/adom.201801190.

22. Li, Y. Pan, R. Xiao, Q. Peng, S. Zhang, D. Ma, F. Li, F. Shen, Y. Wang, B. Yang, Y. Ma. Employing 100\% Excitons in OLEDs by Utilizing a Fluorescent Molecule with Hybridized Local and Charge-Transfer Excited State // Adv. Funct. Mater. 24 (2014) 1609-1614. doi:10.1002/adfm.201301750.

\section{Figures}




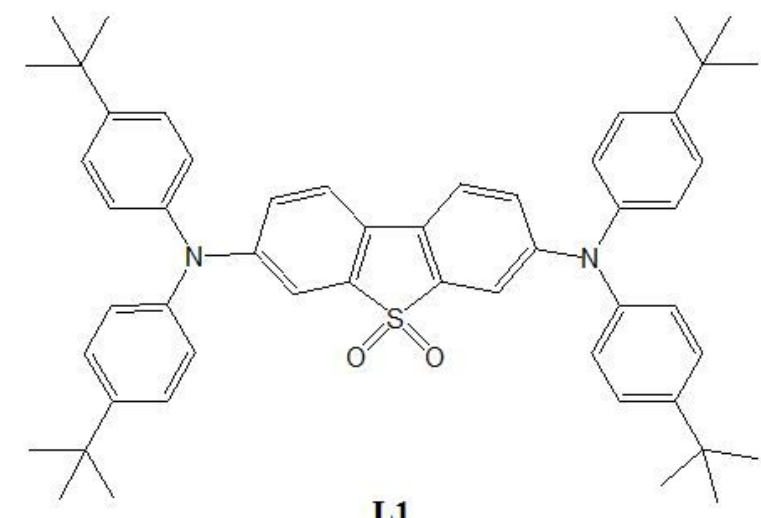

L1

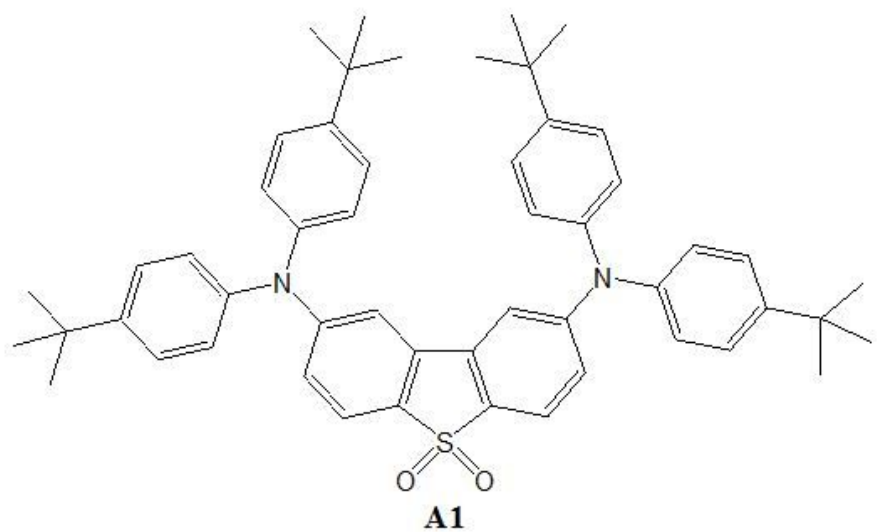

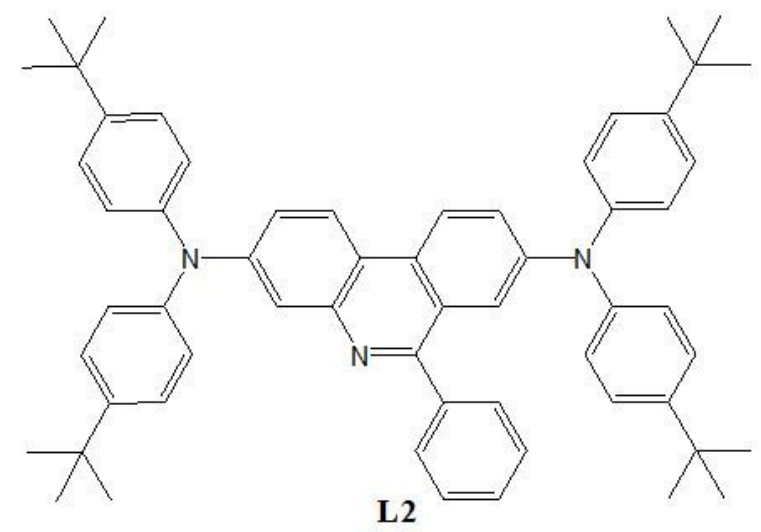

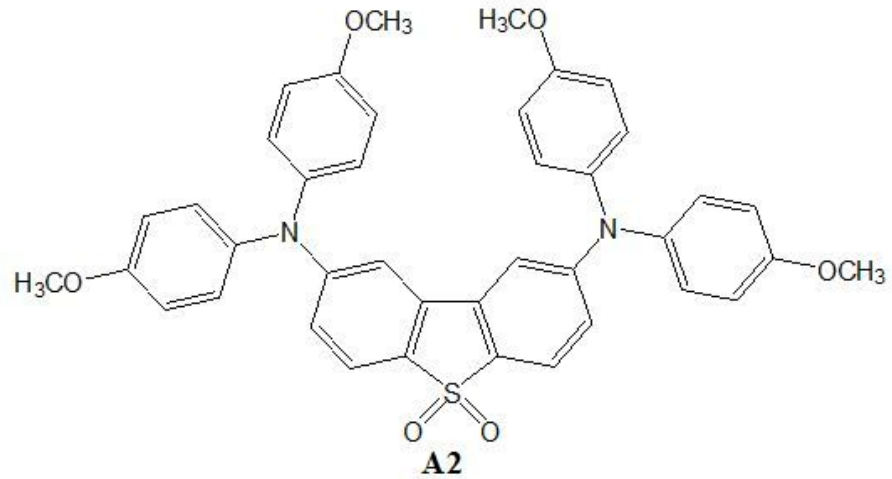

Figure 1

Structural formulas of the studied compounds
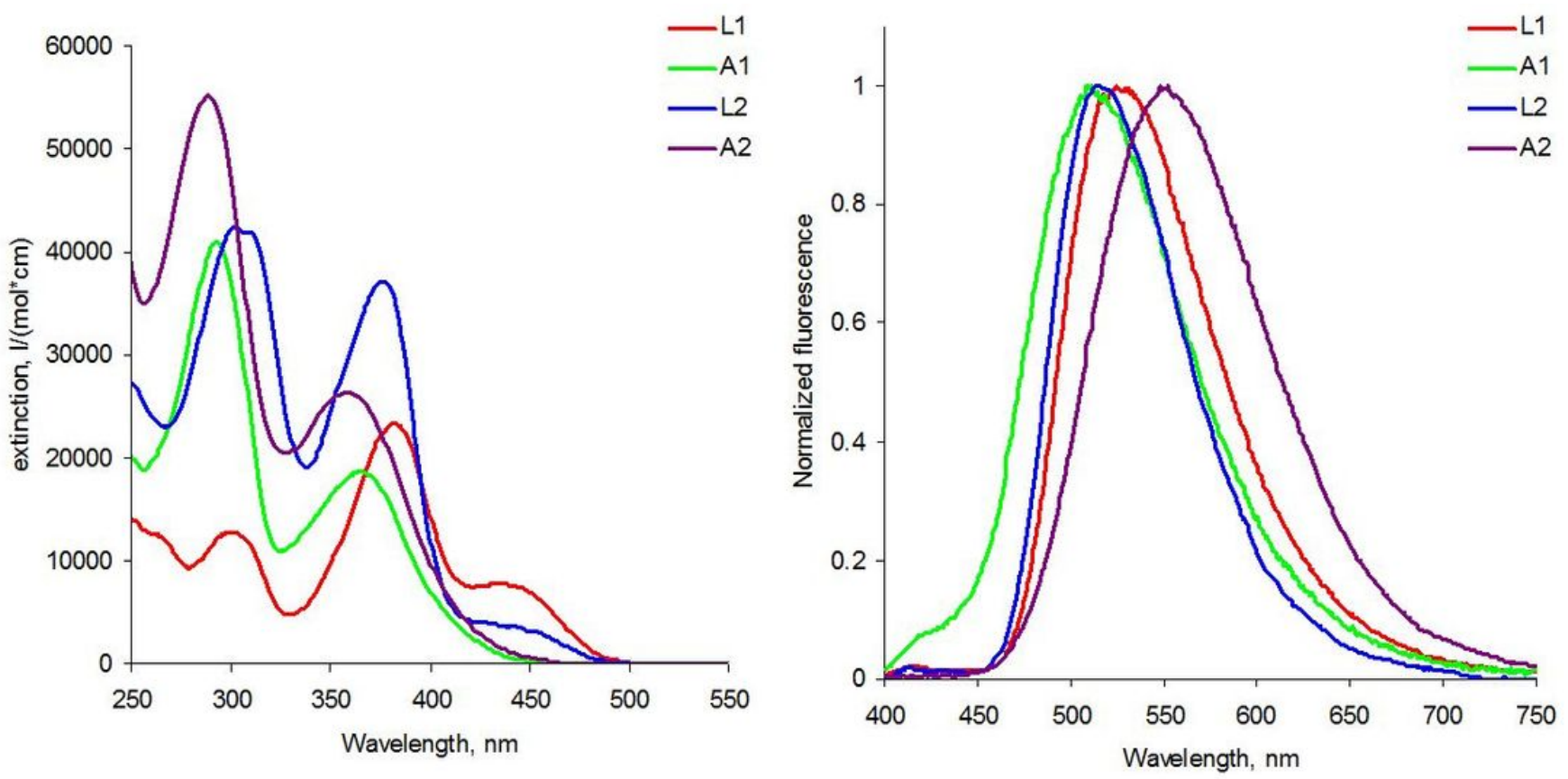

Figure 2

Absorption and fluorescence spectra of solutions of compounds in chloroform 

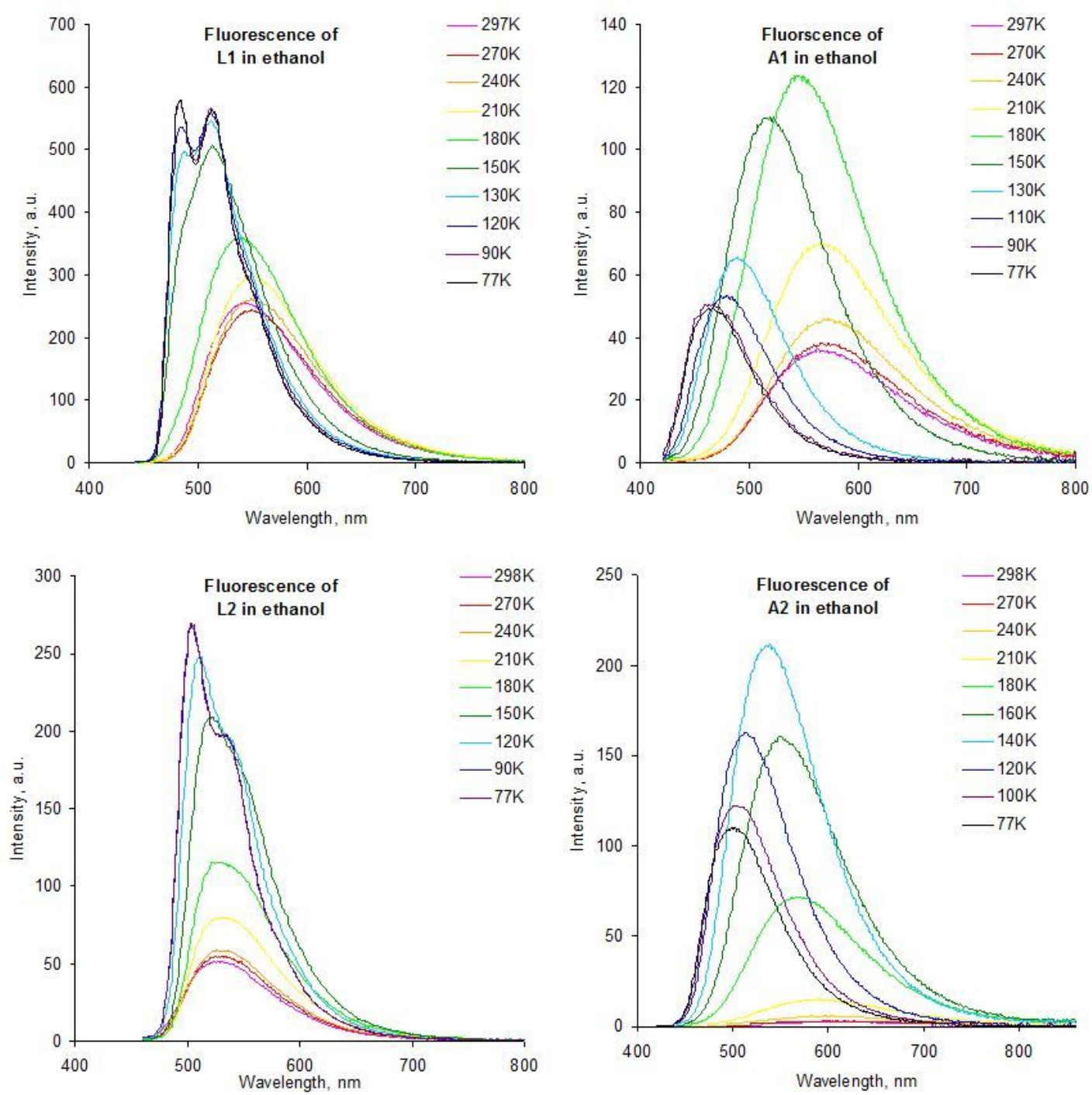

Figure 3

Fluorescence spectra of compounds in ethanol at different temperatures 

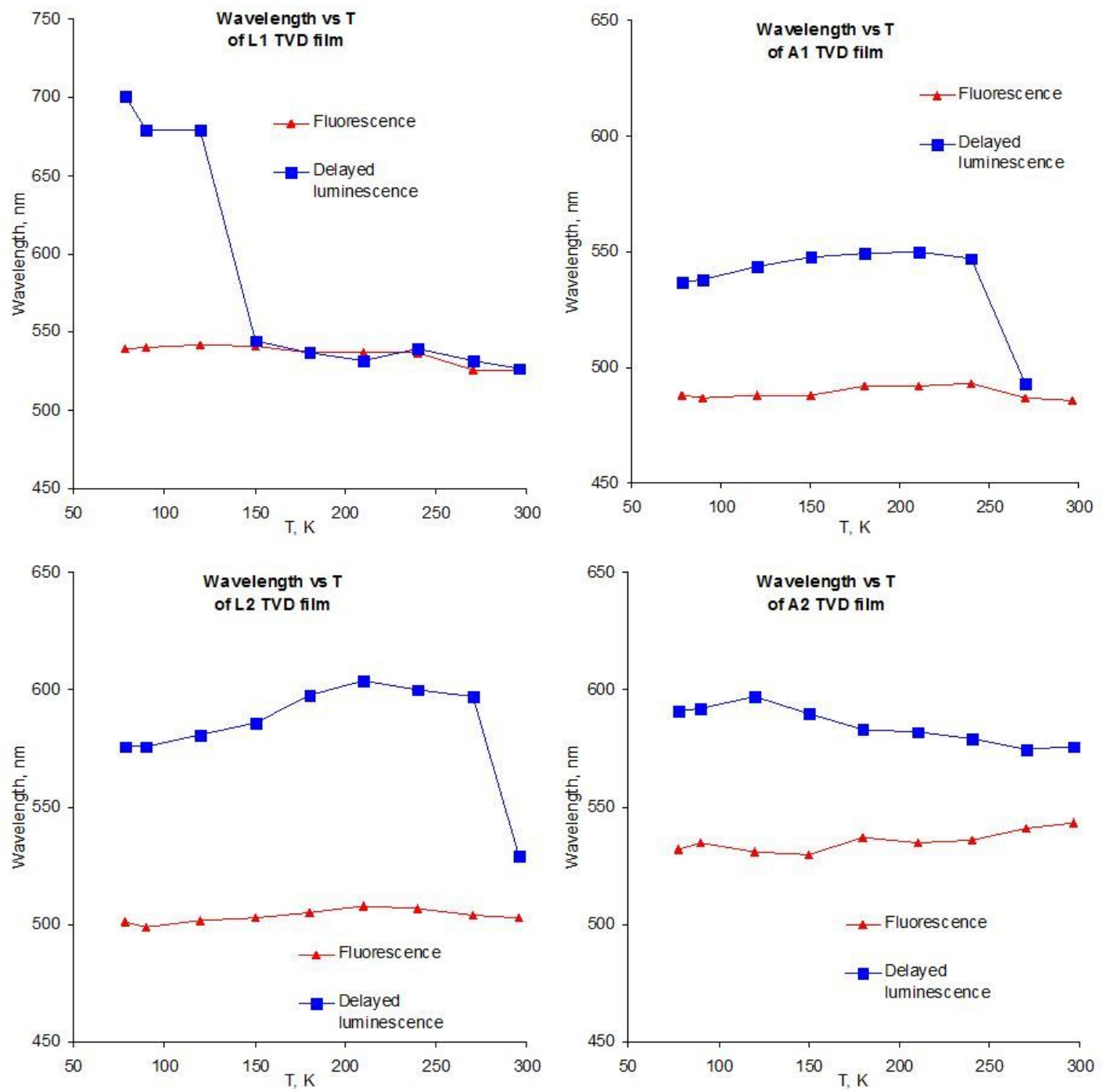

Figure 4

Fluorescence and delayed luminescence maxima position of TVD films at different temperatures 


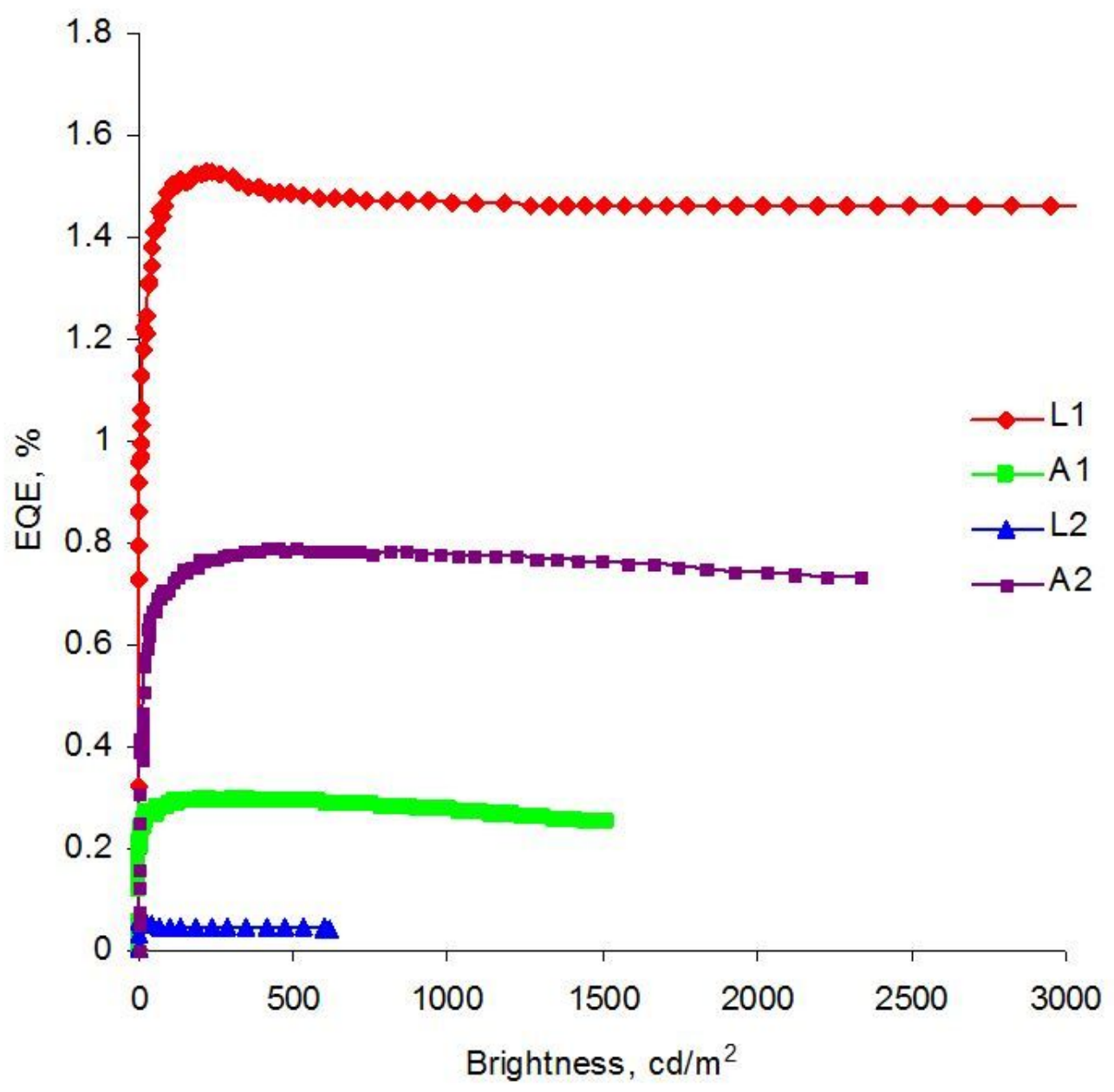

Figure 5

EQE vs brightness for fabricated OLED devices 